\title{
An Efficient Synthesis of Racemic Tolterodine
}

\author{
Joo Hi Kang, Jong Hyoup Lee, ${ }^{\dagger}$ Young Jun Park, ${ }^{+}$Kyoung Soo Kim, ${ }^{\dagger,{ }^{\star *}}$ and Jac Yeol Lee \\ Research Institute for Basic Sciences and Deparment of Chemistry. College of Sciences, Kung Hee Lniversity. \\ Seoul 130-701, Korea. E-mail: ljyäkhu.ack \\ "Chirogenix Co., Lad, Kowoon Instithe of Technology Innovation, Swon Lniversity, Whasung, Kynggi $415-743$, Korea \\ E-mail: kskimpcrá, chirogenix.com \\ Recened Angtist 11. 2007
}

Key Words : Tolterodine, Racemate

Tolterodine, in particular $(-)$ - $(R)$-tolterodine L-tartrate $\left(\right.$ Detrol $\left.^{k}\right)$, is a new and potent competitive muscarinic receptor antagonist and is used to treat urinary incontinence (Figure 1).' The drug acts on $\mathrm{M}_{2}$ and $\mathrm{M}_{3}$ subtypes of muscarinic receptors whereas other antimuscarinic treatment (eg. oxybutynin) for overactive bladder only acts on $\mathrm{M}_{3}$ receptors making them more selective. ${ }^{2-5}$ Nonetheless. tolterodine has fewer side effects than other antimuscarinics because tolterodine targets the bladder more than other areas of the body." "This means that less drug needs to be given daily (due to efficient targeting of the bladder) and so there are fewer side effects. Therefore, some different approaches have been published for racemic and asymmetric synthesis of tolterodine. ${ }^{7 \cdot 14}$ In the case of racemic tolterodine, a classical resolution by the formation of diastereomeric salt using $\mathrm{L}-(+)$-tartaric acid is used to achieve pure $(R)$ tolterodine, and the racemization of $(S)$-tolterodine for the recycle does not seem particularly difficult to accomplish, even if it require additional cost (Figure 1$).^{15}$

Herein we report an efficient synthetic route of racemic tolterodine suitable for the large-scale commercial production by modifying the reported synthetic routes."

The synthetic route to $( \pm$ )-tolterodine (1) proposed by us is depicted in Scheme 1. Commercially available 6-methyl-4chroman-2-one (2) as a starting material was reduced with $\mathrm{LiBH}_{4}$ to afford a diol 3 in $93 \%$ yield, which was treated with 2.2 equiv of $\mathrm{CH}_{3} \mathrm{SO}_{2} \mathrm{Cl}$ in the presence of triethylamine to give a dimesylated compound $\mathbf{4}$ in quantitative yield. A solution of compound $\mathbf{4}$ in acetonitrile was treated with 1.5 equiv of KI followed by an addition of 10.5 equiv of $N, N$ dissopropylamine to provide mono-mesylated amine $\mathbf{5}$ in quantitative yield. Finally, hydrolysis of 5 with $\mathrm{NaOH}$ in

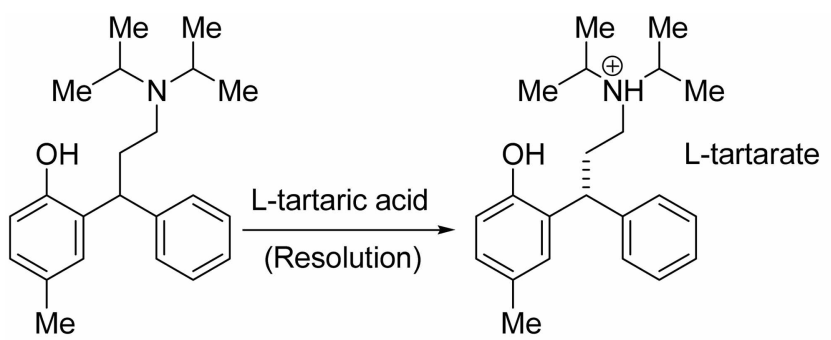

tolterodine (1)
$(+)-(R)$-tolterodine L-tartarate
Figure 1. Resolution ol racemic tolterodinc.
MeOH- $\mathrm{H}_{2} \mathrm{O}$ provided $( \pm)$-tolterodine (1) in quantitative yield.

In summary, the synthesis of $( \pm)$-tolterodine (1), a precursor of $(+)-(R)$-tolterodine, was efficiently performed from 6-methyl-4-chroman-2-one (2) via 4 steps in high yield. This process is suitable for large-scale commercial production by avoiding hazardous reagents and high pressure of hydrogen gas.

\section{Experimental Section}

2-(3-Hydroxy-1-phenylpropyl)-4-methylphenol (3). ']o a solution of 6-methyl-4-chroman-2-one $(2,37.5 \mathrm{~g}, 0.16$<smiles>Cc1ccc2c(c1)C(c1ccccc1)CC(=O)O2</smiles><smiles>COCCC(c1ccccc1)c1cc(C)ccc1OC</smiles>

Scheme 1. Reagents and raction conditions: (a) $\mathrm{LiBH}_{4}(1.0$ cquiv) THF, 0 "C to it. overnight, 93\%: (b) $\mathrm{CH}_{3} \mathrm{SO}_{2} \mathrm{Cl}$ (2.2 equiv). TFA (2.5 equiv), $\mathrm{Cl}_{2} \mathrm{Cl}_{2}, 0^{\circ} \mathrm{C}$. $10 \mathrm{rt}, 1 \mathrm{~h}, 99 \%$, (c) $\mathrm{KI}$ (1.5 equiv), CI;CN. rellux. $6 \mathrm{~b}$. and then $\mathrm{A}$. $\mathrm{A}$-diisopropylamine $(10.5$ equiv), $\mathrm{rt}$ to 70 " $\mathrm{C} .24$ h. $99 \%$ : (d) $\mathrm{NaOH}$ (5.0 equiv), $\mathrm{MeOH} / \mathrm{H}_{2} \mathrm{O}$ (2:1). 85 'C. 10 h. $99 \%$. 
$\mathrm{mol})$ in tetrahydrofuran $(500 \mathrm{~mL})$. was slowly added lithium borohydride $(3.60 \mathrm{~g} .0 .16 \mathrm{~mol})$ at $0{ }^{\circ} \mathrm{C}$. The reaction mixture was stirred at room temperature for $24 \mathrm{~h}$. cooled to $0^{\circ} \mathrm{C}$. and quenched with water $(50 \mathrm{~mL})$. After renoval of solvent. the resulting residue was adjusted to $\mathrm{pH} \mathrm{l}$ with water $(150 \mathrm{~mL})$ and conc. $\mathrm{HCl}(20 \mathrm{~mL})$. The mixture was extracted with ethyl acetate. The extract was washed with water, dried over $\mathrm{MgSO}_{4}$, and concentrated in vacto to afford 3 as a white solid (35.4 g. $93 \%$ ): nip $111-113^{\circ} \mathrm{C}$; ${ }^{1} \mathrm{H}$ NMR $(400 \mathrm{MHz}$. $\left.\mathrm{CDCl}_{3}\right) \delta 7.29-7.18(\mathrm{~m} .5 \mathrm{H}$ ). 6.84 (dd. $J=8.1,1.9 \mathrm{~Hz}, \mathrm{lH}$ ). $6.76(\mathrm{~d} . J=1.7 \mathrm{~Hz} . \mathrm{HH}), 6.70(\mathrm{~d}, J=8.1 \mathrm{~Hz}, \mathrm{lH}) .4 .56(\mathrm{~m}$. lH). 3.82 (br S. $2 \mathrm{H}) .3 .72(\mathrm{~m}, \mathrm{lH}) .3 .52(\mathrm{~m} . \mathrm{lH}) .2 .36(\mathrm{~m}$. $1 \mathrm{H}), 2.17(\mathrm{~s}, 3 \mathrm{H}), 2.15(\mathrm{~m}, 1 \mathrm{H}):{ }^{13} \mathrm{C}$ NMR $(100 \mathrm{MHz}$. $\left.\mathrm{CDCl}_{3}\right) \delta$ 151.4. 144.0. 130.5, 130.2, 129.1, 128.4. 128.2. 127.9. 126.3. 116.0. 60.7,38.7,37.0, 20.7.

2-(3-Methanesulfonyloxy-1-phenyl-propyl)-4-methylphenyl methanesulfonate ( 4$)$. To a solution of diol 3 (18.3 g. $75.6 \mathrm{mmol})$ in $\mathrm{CH}_{2} \mathrm{Cl}_{2}(300 \mathrm{~mL})$ was added triethy lamine (26.4 mL. 189.0 munol. 2.5 equiv) and methanesulfonyl chloride ( $12.9 \mathrm{~mL}, 166.0 \mathrm{mmol}, 2.2$ equiv.) at $0{ }^{\circ} \mathrm{C}$. After stirring at room tentperature for $1 \mathrm{~h}$. the resulting mixture was washed with water $(100 \mathrm{~mL})$ and dried over $\mathrm{MgSO}_{4}$. and concentrated in wonio to provide $4(29.7 \mathrm{~g} .99 \%)$ as a brown oil: ${ }^{1} \mathrm{H} \mathrm{NMR}\left(400 \mathrm{MHz}_{2} \mathrm{CDCl}_{3}\right) \delta 7.32-7.22(\mathrm{~m}, 7 \mathrm{H})$. $7.12($ d. $J=1.9 \mathrm{~Hz}, 1 \mathrm{H}) .7 .05($ dd. $J=8.3,1.8 \mathrm{~Hz}, 1 \mathrm{H}), 4.56$ (t. $J=7.8 \mathrm{~Hz} .1 \mathrm{H}), 4.2 \mathrm{l}-4.16$ (m. $2 \mathrm{H}$ ). 3.01 (s. $3 \mathrm{H}$ ). 2.93 (s. $3 \mathrm{H}), 2.47(\mathrm{~m}, 2 \mathrm{H}), 2.33(\mathrm{~s}, 3 \mathrm{H}):{ }^{13} \mathrm{C}$ NMR $(100 \mathrm{MHz}$. $\left.\mathrm{CDCl}_{3}\right) \delta 145.0 .141 .8 .137 .4,135.7,129.2,128.8 .128 .7$. 128.1. $127.0,121.6 .68 .0,39.8 .37 .9,37.3 .34 .3,21.2$.

2-(3-N,N-Diisopropylamino-1-phenylpropyl)-4-methylphenyl methanesulfonate (5). A reaction mixture of $\mathbf{4}(29.7$ g. $74.5 \mathrm{mmol}$ ) and potassium iodide (18.6 g. $111.8 \mathrm{mmol}$. 1.5 equiv.) in acetonitrile $(500 \mathrm{~mL})$ was heated at reflux for 6 $\mathrm{h}$. The reaction mixture was cooled to room temperature and treated with $N, N$-diisopropy lanine $(109.7 \mathrm{~mL}, 782.6 \mathrm{mmol}$. 10.5 equiv). The resulting mixture was further stirred at reflux for $24 \mathrm{~h}$. After cooling to room temperature. the organic solvent was removed under reduced pressure. The residue was extracted with ethyl acetate. The combined organic extract was washed with water dried over $\mathrm{MgSO}_{4}$. and concentrated in vacto to afford $5(30.0$ g. $99 \%)$ as a yellowish oil: ${ }^{1} \mathrm{H}$ NMR ( $\left.400 \mathrm{MHz} . \mathrm{CDCl}_{\hat{\jmath}}\right) \delta 7.30-7.22(\mathrm{~m}$. $6 \mathrm{H}), 7.14(\mathrm{~m} . \mathrm{HH}), 6.99(\mathrm{dd} . J=8.4 .1 .9 \mathrm{~Hz} .1 \mathrm{H}), 4.35(\mathrm{t} . J=$ $7.5 \mathrm{~Hz} .1 \mathrm{H}$ ). $3.00-2.93$ (m. 2H). 2.77 (s. $3 \mathrm{H}) .2 .39-2.33$ (m. $2 \mathrm{H}) .2 .30(\mathrm{~s} .3 \mathrm{H}) .2 .15-2.09(\mathrm{~m} .2 \mathrm{H}) .0 .93-0.91(\mathrm{~m} .12 \mathrm{H})$. ${ }^{13} \mathrm{C} \mathrm{NMR}\left(100 \mathrm{MHz}, \mathrm{CDCl}_{5}\right) \delta: 145.6,143.9,137.2,136.9$. 129.4. 128.5. 128.2, 128.1, 126.3, 121.5. 120.9. 48.8, 43.7. 41.9 .37 .7 .37 .4 .37 .3 .20 .8 .20 .6 .
(土)-Tolterodine: 2-[3-( $N, N$-diisopropylamino)-1-pheny]propyl]-t-methylphenol (1). To a solution of $5(51.74 \mathrm{~g}$. $0.13 \mathrm{~mol})$ in mixture of $\mathrm{CH}_{2} \mathrm{OH}$ and $\mathrm{H}_{2} \mathrm{O}(2: 1.500 \mathrm{~mL})$ was added sodium hydroxide ( $25.6 \mathrm{~g}, 0.64 \mathrm{~mol} .5 .0$ equiv.). The reaction mixture was heated at $85^{\circ} \mathrm{C}$ for $10 \mathrm{~h}$ and cooled to room temperature. The volume of reaction mixture was reduced to $1 / 3$ under reduced pressure. The reaction mixture was adjusted to $\mathrm{pH} 8$ with conc. $\mathrm{HCl}$ and extracted with ethyl acetate. The organic layer was dried over $\mathrm{MgSO}_{4}$ and concentrated in vocto to give ( \pm )-tolterodine $(\mathbf{1 .} 40.0 \mathrm{~g}$. $99 \%$ ) as an oil: ${ }^{1} \mathrm{H}$ NMR $\left(400 \mathrm{MHz}, \mathrm{CDCl}_{3}\right) \delta 7.32-7.18(\mathrm{~m}$. $5 \mathrm{H}) .6 .81-6.78(\mathrm{~m}, 2 \mathrm{H}) .6 .60(\mathrm{~m} .1 \mathrm{H}), 4.47(\mathrm{dd}, J=11.1$, $4.18 \mathrm{~Hz} . \mathrm{lH}), 3.25-3.18(\mathrm{~m}, 2 \mathrm{H}) .2 .69(\mathrm{~m}, \mathrm{lH}) .2 .38-2.36$ $(\mathrm{m}, 2 \mathrm{H}), 2.12(\mathrm{~m}, \mathrm{lH}) .2 .11(\mathrm{~s}, 3 \mathrm{H}) .1 .11(\mathrm{~d} . J=6.7 \mathrm{~Hz} .6 \mathrm{H})$. $1.06(\mathrm{~d}, J=6.7 \mathrm{~Hz} .6 \mathrm{H}):{ }^{13} \mathrm{C}$ NMR $\left(100 \mathrm{MHz} . \mathrm{CDCl}_{3}\right) \delta$ : $153.1,144.7,132.2$. 129.2. 128.7, $128.5,128.3 .127 .8$. $126.1,117.9,48.5 .42 .6,39.7 .33 .4,20.8$. 19.9, 19.5 .

Acknowledgments. This work was supported by the Small and Medium Business Administration, Korea.

\section{References}

1. Jonlas. U.: Hoefner. K.: Madersbacher. H.: Holmdahl. T. H. Hortd J. Lrol. 1997. 15. 144.

2. Gillberg. P. G.; Sundquist, S.; Nilvebrant, L. Ew: J. Pharmacol. 1998. $3+9,285$.

3. Yamanishi. T.: Chapple, C. R.: Chess-Williams. R. World J. Grol. 2001. 19. 299.

4. Sellers. D. I.: Yamanishi. I.: Chapple. C. R.: Couldwell. C.: Yasuda. K.: Chess-Williams. R. J. Anton. Phomocol 2000. 20. 171 .

5. Hirose, H; Aoki. I; Kimura. T.: Fujikawa, T.; Numazawa, T:; Sasaki, K.: Nishikibe. M: Noguchi. K. Ew: J. Pharmacol 2002. +52.245.

6. Nilvebrant. L.: Andersson. K. E.: Gillberg. P. G.: Stahl. M.: Sparf. B. Eur. J. Pharmacol 1997.327. 195.

7. Andersson, P. G.; Schink, H. E.: Österlund. K. J. Org. Chem. 1998. 63,8067 .

8. Gage. J. R.: Cabaj. J. E. L.S. Patent 5.922.914. 1999.

9. Botteghi. C.: Corrias. T.: Marchetti. M.: Paganelli. S.: Piccolo. O. Org. Process Res. Dew 2002.6 .379$.

10. Selensky. C.: Pettus. T. R. R. J. Org. Chent 2004. 69.9196.

11. Colombo. L.: Rossi. R.: Castaldi. G.; Allegrini, P.; Dipharma, S. P. A. Italian Patent 1/12004.4000616, 2004: Canadian Patent CA 2502640. 2005; European Patent EP 158+621, 2005.

12. Hedberg. C.: Andersson. P. G. Adh: Simth. Catal 2005. 347. 662.

13. Chen1. G.: Tokun1aga. N.: Hayashi. I. Org. Lett. 2005. 7. 2285.

14. Ulgheri. F.: Marchetti. M.: Piccolo. O. J. Org. Chent. 2007. 72. 6056 .

15. Srinivas, K; Srinivasan. N.: Reddy. K. S; Ramakrishna, M; Reddy, C. R.: Arunagiri, M: Kumari. R. L: Venkataraman, S.; Mathad. V. T. Org. Process Res. Der: 2005. 9.314 